BULLETIN OF THE

AMERICAN MATHEMATICAL SOCIETY

Volume 80, Number 5, September 1974

\title{
MEASURABLE CHOICE AND THE INVARIANT SUBSPACE PROBLEM
}

\author{
BY EDWARD A. AZOFF AND FRANK GILFEATHER ${ }^{1}$
}

Communicated by P. R. Halmos, February 8, 1974

In [1], J. Dyer, A. Pedersen and P. Porcelli announced that an affirmative answer to the invariant subspace problem would imply that every reductive operator is normal. Their argument, outlined in [1], provides a striking application of direct integral theory. Moreover, this method leads to a general decomposition theory for reductive algebras which in turn illuminates the close relationship between the transitive and reductive algebra problems.

The main purpose of the present note is to provide a short proof of the technical portion of [1]: that invariant subspaces for the direct integrands of a decomposable operator can be assembled "in a measurable fashion". The general decomposition theory alluded to above will be developed elsewhere in a joint work with C. K. Fong, though we do present a summary of some of its consequences below.

All Hilbert spaces discussed in this paper will be separable and all operators will be bounded. We use the term 'algebra' to refer to an identity-containing algebra of operators which is closed in the weak operator topology. A transitive algebra is an algebra having no nontrivial invariant subspaces; more generally, an algebra is called reductive if it is reduced by each of its invariant subspaces.

The reader is referred to [2] or [3] for the details of direct integral theory; the primary purpose of the following summary is to fix notation. Let $\mu$ be the completion of a finite positive regular Borel measure supported on a $\sigma$-compact subset of a separable metric space $\Lambda$ and let $\left\{e_{n}\right\}, 1 \leqq n \leqq \infty$, be a collection of disjoint Borel subsets of $\Lambda$ with union $\Lambda$. Let $h_{1} \subseteq h_{2} \subseteq \cdots$ $\subseteq h_{\infty}$ be a sequence of Hilbert spaces with $h_{n}$ having dimension $n$ and $h_{\infty}$ spanned by the remaining $h_{n}$ 's. We write $h=\int_{\Lambda} \oplus h(\lambda) \mu(d \lambda)$ for the Hilbert space of (equivalence classes of) weakly measurable functions $f$ from $\Lambda$ into $h_{\infty}$ such that for $\lambda \in e_{n}, f(\lambda) \in h(\lambda) \equiv h_{n}$, and $\int_{\Lambda}\|f(\lambda)\|^{2} \mu(d \lambda)<\infty$. The element in $h$ represented by the function $\lambda \rightarrow f(\lambda)$ is denoted by $\int_{\Lambda} \oplus f(\lambda) \mu(d \lambda)$.

AMS (MOS) subject classifications (1970). Primary 47A15, 46L15; Secondary $28 \mathrm{~A} 20$.

${ }^{1}$ The first author was supported by NSF grant GP 38488 and the second by NSF grant GP 31435.

Copyright (C) American Mathematical Society 1974 
An operator $A$ on $h$ is said to be decomposable if there exists a $\mu$-measurable operator-valued function $A(\cdot)$ defined on $\Lambda$ for which $A(\lambda)$ is an operator on $h(\lambda)$ and for $f$ in $h,(A f)(\lambda)=A(\lambda) f(\lambda)$. We write $A=$ $\int_{\Lambda} \oplus A(\lambda) \mu(d \lambda)$ for the equivalence class corresponding to $A(\cdot)$. If $A(\lambda)$ is a scalar multiple of the identity on $h(\lambda)$ for almost all $\lambda$, then $A$ is called diagonal.

The following proposition should be compared with [1, Theorem 3(a)]. The measure-theoretic difficulties alluded to there are avoided here because the hypotheses on $\Lambda$ allow us to use von Neumann's principle of measurable choice.

Proposition. Let $A=\int_{\Lambda} \oplus A(\lambda) \mu(d \lambda)$ be a decomposable operator on $h$. Then the set $T$ of $\lambda \in \Lambda$, for which $A(\lambda)$ has a nontrivial invariant subspace, is measurable. Furthermore, there exists a decomposable projection $P=\int_{\Lambda} \oplus P(\lambda) \mu(d \lambda)$ such that $P h$ is invariant under $A$ and $P(\lambda)$ is nontrivial if and only if $\lambda \in T$.

Proof. Without loss of generality, we assume that $\Lambda=e_{k}$ for some $k \leqq \infty$ and that $\|A\| \leqq 1$. We denote by $B$ the set of contraction operators on $h_{k}$ with the weak operator topology and recall that this makes $B$ into a compact metric space. Thus, by modifying the $\{A(\lambda)\}$ on a set of measure zero if necessary, we may as well assume the correspondence $\lambda \rightarrow A(\lambda)$ is a Borel map between $\Lambda$ and $B$.

Set $E=\{(\lambda, Q) \in \Lambda \times B: Q$ is a nontrivial projection and $Q A(\lambda) Q=$ $A(\lambda) Q\}$. Then $\Pi_{\Lambda}(E)=T$ and since composition is a Borel map from $B \times B$ to $B$, we see that $E$ is a Borel subset of $\Lambda \times B$. We now apply von Neumann's principle of measurable choice ([2, Chapter I, Lemma 4.7] or $[3, \S 16$, Lemma 4]). This shows that $T$ is measurable and gives us a Borel function $P$ whose domain $D$ differs from $T$ by a set of measure zero and whose graph is contained in $E$. The proof is completed by setting $P(\lambda)=0$ for $\lambda \notin D$.

The following is the result announced in the title of [1].

THEOREM. The following are equivalent:

(1) Every operator on a Hilbert space of dimension greater than one has a nontrivial invariant subspace.

(2) Every reductive operator is normal.

Proof. The implication (2) implies (1) is clear. Conversely, assume (1) and suppose $A$ is a reductive operator on a Hilbert space $h$. Let $\mathscr{E}$ be a maximal abelian von Neumann subalgebra of $\{A\}^{\prime}$ (the commutant of $A$ ). Applying Theorem 6 of [2, Chapter I], we decompose $h$ as a direct integral, $h=\int_{\Lambda} \oplus h(\lambda) \mu(d \lambda)$, of Hilbert spaces in such a way that $\mathscr{E}$ coincides with the corresponding algebra of diagonal operators. Since $A$ commutes with $\mathscr{E}$, it is decomposable and we write $A=\int_{\Lambda} \oplus A(\lambda) \mu(d \lambda)$. 
Let $P$ and $T$ be as in the Proposition. Clearly $P$ commutes with $\mathscr{E}$ and since $A$ is reductive, $P$ also commutes with $A$. Thus the maximality of $\mathscr{E}$ shows $P \in \mathscr{E}$, i.e. $P(\lambda)$ is trivial for almost all $\lambda$. Consequently, $T$ has measure zero and it follows from (1) that almost all of the spaces $h(\lambda)$ are one dimensional. In particular $A=\int_{\Lambda} \oplus A(\lambda) \mu(d \lambda)$ is diagonal and hence normal.

The technique illustrated above can be used to develop a method for expressing a reductive algebra as a direct integral of transitive algebras. Naturally this illuminates the close relationship between the transitive and reductive algebra problems. The following three results (as well as the one above) all follow with the aid of such a decomposition and illustrate this point.

THEOREM. The following are equivalent:

(1) The only abelian transitive algebra is the trivial one.

(2) Every abelian reductive algebra is selfadjoint.

THEOREM. The following are equivalent:

(1) Every operator $A$ has a nontrivial hyperinvariant subspace (i.e. invariant for $\left.\{A\}^{\prime}\right)$ or is a multiple of the identity.

(2) Every reductive algebra of the form $\{A\}^{\prime}$ is selfadjoint.

THEOREM (RADJAVI-ROSENTHAL). Every reductive algebra containing a maximal abelian selfadjoint algebra of operators is selfadjoint.

\section{REFERENCES}

1. J. A. Dyer, E. A. Pedersen and P. Porcelli, An equivalent formulation of the invariant subspace conjecture, Bull. Amer. Math. Soc. 78 (1972), 1020-1023. MR 46 \#6068.

2. J. T. Schwartz, $W^{*}$-algebras, Gordon and Breach, New York, 1967. MR 38 \#547.

3. J. von Neumann, On rings of operators. Reduction theory, Ann. of Math. (2) 50 (1949), 401-485. MR 10, 548.

Department of Mathematics, University of Georgia, Athens, Georgia 30602

Department of Mathematics, University of Nebraska, Lincoln, Nebraska 68508

Current address (Edward A. Azoff): Department of Mathematics, University of Iowa, Iowa City, Iowa 52240 